\title{
Modelling the mechanical behaviour of expansive clays
}

\author{
E.E. Alonso *, J. Vaunat, A. Gens \\ UPC-DIT, c/Jordi Girona 1-3, Módulo D-2, 08034 Barcelona, Spain
}

\begin{abstract}
A simple formalism is presented to model the behaviour of expansive clays. Two levels of structure are considered. The behaviour of the macrostructure follows the model developed for unsaturated materials by Alonso et al. [Géotechnique 40 (3) (1990) 405-430]. The behaviour of the microstructure is adapted from the work of Gens and Alonso [Can. Geotech. J. 29 (1992) 1013-1032] in order to include the possibility of the micropores being partially saturated. Mechanical coupling between both levels of structure are defined through two functions, one for wetting and the other for drying. They express the change in macrostructural void ratio due to a change in microstructural void ratio, and their value depends on the state of compaction of the macrostructure. The general shape of these curves is discussed on the basis of experimental evidence. Phenomena such as the dependency of strain on stresssuction path, accumulation of expansion strain during suction cycles at low confining stress, accumulation of compression strain during suction cycles at high confining stress, strain fatigue during drying-wetting cycles, macropore invasion by expanded microstructure and development of macroporosity during strong drying can be represented. A mathematical formulation of the model is described and its performance finally assessed by comparison with laboratory tests. (C) 1999 Elsevier Science B.V. All rights reserved.
\end{abstract}

Keywords: Elasticity; Expansive soils and rocks; Model; Plasticity; Suction; Swelling

\section{Introduction}

The mechanical behaviour of compacted expansive clays is a critical factor in the design of seals for repositories to store hazardous wastes. An adequate constitutive model for this type of material is therefore required when realistic numerical simulations of sealing systems are to be performed. The clays are generally compacted and are consequently initially in unsaturated state.

Alonso et al. (1987, 1990) presented the Barcelona Basic Model (BBM) describing the stress-strain behaviour of partially saturated soils

\footnotetext{
* Corresponding author. Fax: + 34-3-401-6504.

E-mail addresses: alonso@etseccpb.upc.es (E.E. Alonso), vvaunat@etseccpb.upc.es (J. Vaunat), gens@etseccpb.upc.es (A. Gens)
}

of low activity. Formulated in the framework of hardening elastoplasticity, this model extends the modified Cam-Clay model (Roscoe and Burland, 1968 ) by considering two independent sets of stress variables: the net stress defined as the excess of total stress over air pressure and the suction. A key element of the model is the dependency of the yield locus on suction, which can be represented in the $p-q-s$ diagram ( $p=$ mean net stress, $q=$ deviatoric stress and $s=$ suction) by the LC surface (Fig. 1). BBM is able to reproduce most responses of partially saturated soils under simple loadingunloading and wetting-drying paths. These are:

- increase of shear strength and preconsolidation pressure $p_{0}$ with suction;

- development of reversible swelling strains when suction decreases at low confining stress; 


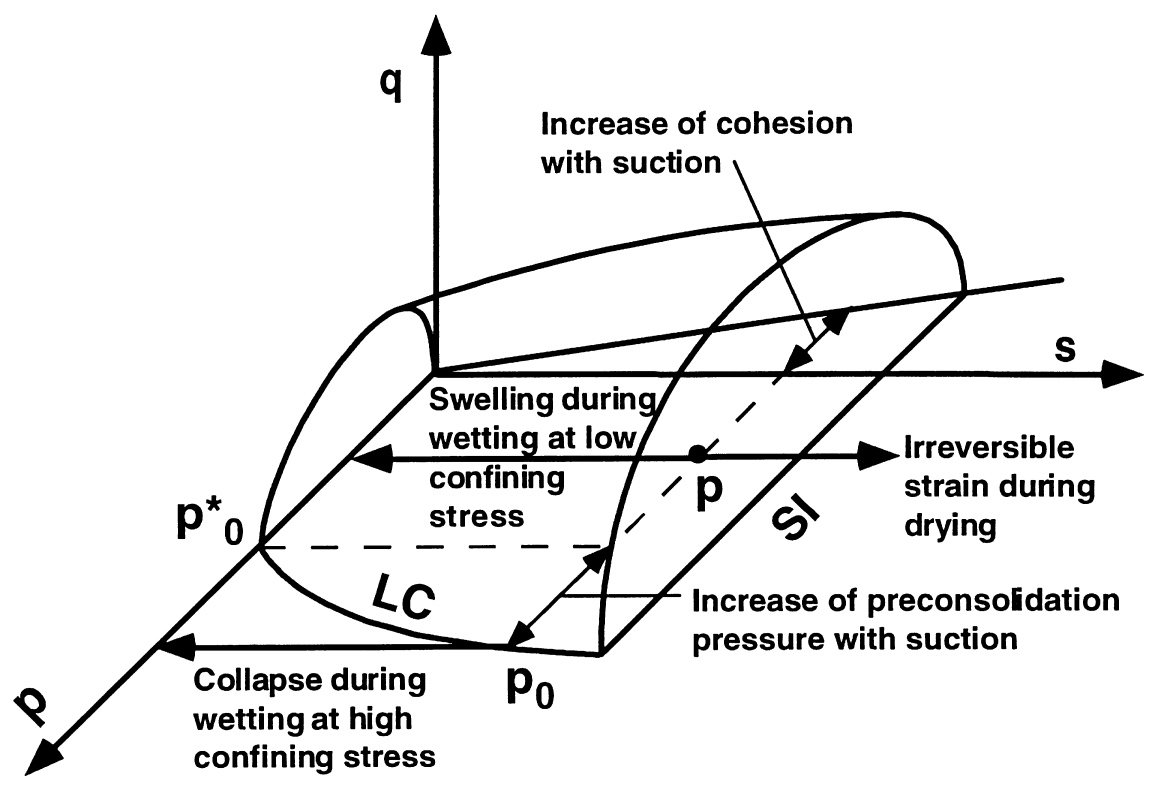

Fig. 1. BBM yield locus in $p-q-s$ plane.

- occurrence of irreversible collapse strains when suction decreases at high confining stress;

- development of irreversible shrinkage when suction increases above a threshold value.

To refer to more specific stress-strain paths involved during the hydration of an engineered barrier, this model gives an explanation of the peak observed during a swelling pressure test performed at a low density as a consequence of the plastification of the sample at constant volume (Fig. 2).

BBM presents, however, limitations in modelling the behaviour of more expansive soils. Particularly, the dependency of swelling strains and swelling pressures on the initial state (Brackley, 1973) and on the stress path (Justo et al., 1984), strain accumulation during suction cycles (Pousada, 1984; Dif and Bluemel, 1991) as well as secondary swelling (Komornik and Zeitlen, 1965) cannot be reproduced by the model. It is believed that such behavioural features are mainly related to the existence of coupled chemo-hydromechanical phenomena between distinct levels of structure within the material. In this paper, an enhanced model, called the Barcelona Expansive Model (BExM) is presented. It is based on the consideration of two levels of structure. The conceptual basis of the model and the associated experimental evidence are first described. A mathematical formulation is afterwards developed and model performances highlighted. The behaviour of expansive soil under load and suction changes as well as suction cycles is considered. Time dependent effects will not be dealt with in the present work.

\section{Microstructural and macrostructural behaviour}

Gens and Alonso (1992) presented a conceptual basis for a model for expansive soils. Two distinct levels are distinguished: the microstructural level at which swelling of active minerals takes place and the macrostructural level responsible for major structural rearrangement. The following assumptions are adopted:

- the microstructural level is mainly saturated and the effective stress concept holds;

- the microstructural behaviour is elastic and volumetric;

- mechanical, hydraulic and chemical equilibrium 


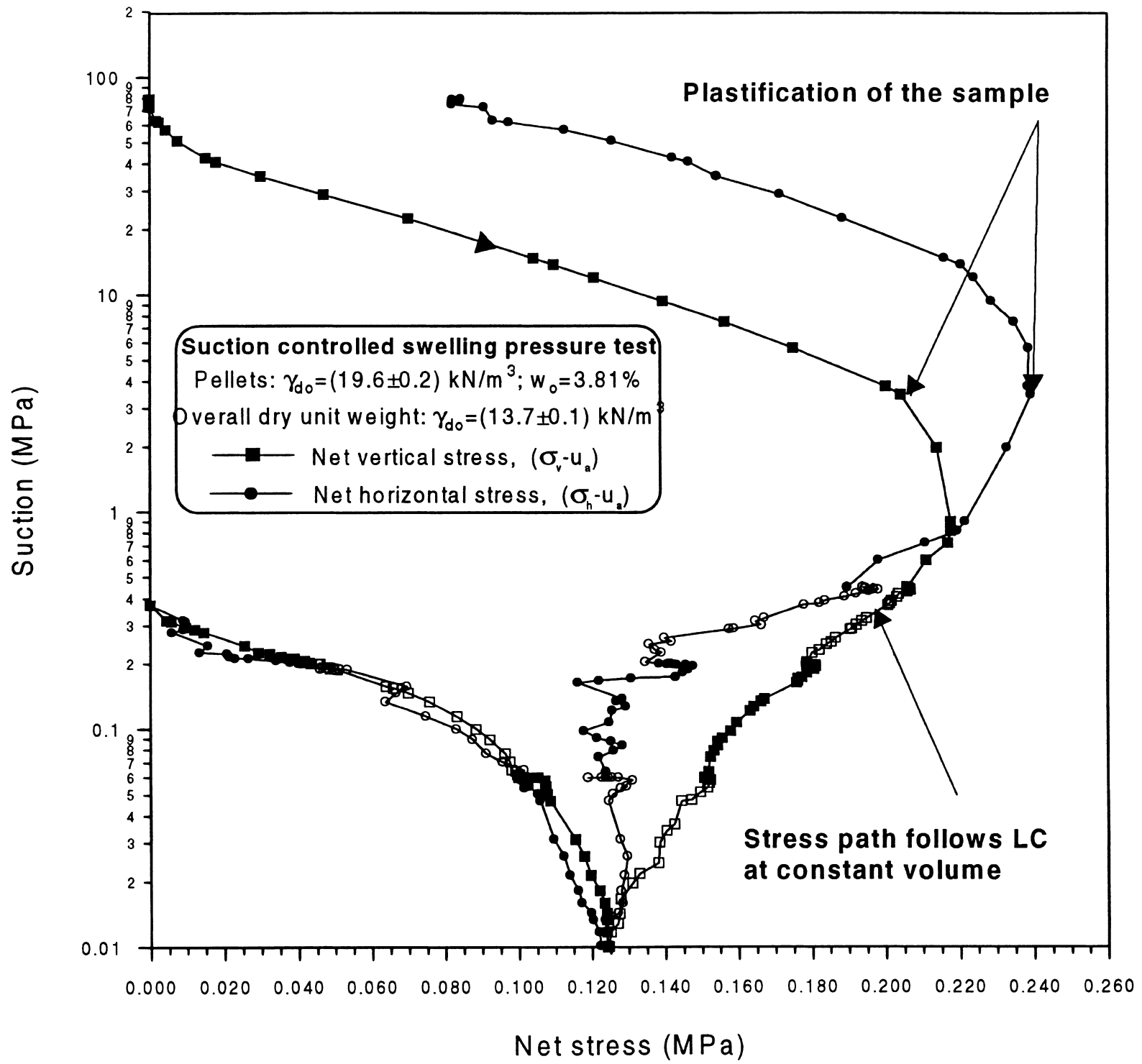

Fig. 2. Swelling pressure test on compacted Boom clay (after Romero, 1999).

exists between microstructure and macrostructure;

- coupling between microstructure and macrostructure results in possible build-up of macrostructural elastoplastic strains when elastic microstructural strains take place.

With these assumptions, the increment of volumetric microstructural strain $\mathrm{d} \epsilon_{\mathrm{vm}}^{\mathrm{e}}$ depends only on the increment of mean effective stress $\mathrm{d} p^{\prime}=$ $\mathrm{d}\left(p-p_{\mathrm{w}}\right)=\mathrm{d}\left(p-p_{\mathrm{a}}+p_{\mathrm{a}}-p_{\mathrm{w}}\right)=\mathrm{d}(p+s)$. Therefore, a straight line $p+s=$ constant can be drawn from the current state $C$ of stress and suction along which no microstructural strain takes place. This line, called Neutral Line (NL), moves with $C$ and separates at each instant the zone of microstructural swelling from the zone of microstructural shrinkage in the $p-s$ plane (see Fig. 3). The authors pointed out, however, that, depending on 


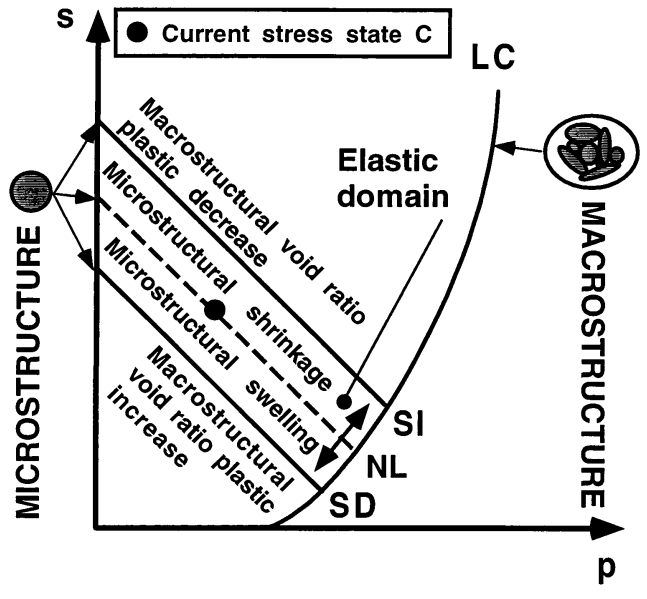

Fig. 3. BExM yield loci in $p-s$ plane.

the type of microstructural expansion of the material, the inclination of NL may diverge from the value of $45^{\circ}$ in some stress-suction regions.

Microstructural swelling affects the structural arrangement of the macrostructure, inducing an irreversible increase of the void ratio $e$. Reciprocally, microstructural shrinkage induces irreversible decrease of $e$. In order to represent such phenomena, two additional yield loci parallel to NL are introduced in the model (Fig. 3). Suction Increase (SI) and Suction Decrease (SD) lines define the onset of macrostructural volumetric plastic strains due, respectively, to microstructural shrinkage and swelling. They bound the elastic domain of the microstructure and become active when respectively touched by NL.

Experimental data evidence a dependency of the macrostructural plastic strain not only on the microstructural strain, but also on the level of stress (Justo et al., 1984) and on the compaction state of the macrostructure (expressed by the void ratio; Brackley, 1973). Alonso et al. (1994) then proposed expressing the increment of the volumetric macrostructural plastic strain due to microstructural swelling/shrinkage by:

$\mathrm{d} \epsilon_{\mathrm{vM}}^{\mathrm{p}}=f_{\mathrm{D}} \mathrm{d} \epsilon_{\mathrm{vm}}^{\mathrm{e}} \quad$ when

$\mathrm{SD}$ is activated

$\mathrm{d} \epsilon_{\mathrm{vM}}^{\mathrm{p}}=f_{\mathrm{I}} \mathrm{d} \epsilon_{\mathrm{vm}}^{\mathrm{e}} \quad$ when SI is activated

where $f_{\mathrm{D}}$ and $f_{\mathrm{I}}$ are two coupling functions which depend on the ratio of $p$ over $p_{0}$ in the case of an isotropic state of stress. $p / p_{0}$ is a measure of the distance from the current stress state to the yield locus of the macrostructure LC and has the same meaning as the overconsolidation ratio for an isotropically consolidated saturated soil.

The macrostructural plastic change in volume attests in turn to the location of the LC. When the macrostructure becomes looser, the macrostructural yield surface shrinks. When the structure becomes denser, the elastic domain increases and LC expands. A coupling then exists between yield surfaces LC, SI and SD. The scope of this paper is to provide further insight into the validity of such coupling, and to give more accurate expressions for the functions $f_{\mathrm{I}}$ and $f_{\mathrm{D}}$.

\section{Interaction between micro- and macrostructure}

\subsection{Wetting and drying paths}

Pousada (1984) performed cyclic suction-controlled oedometer tests on a compacted expansive clay from Madrid, Spain under different vertical applied stress. The physical properties of the material are: $w_{\mathrm{L}}=71 \%, w_{\mathrm{P}}=30 \%, w_{\mathrm{opt}}=33 \%$ and $\gamma_{\mathrm{D}}=13.4 \mathrm{kN} / \mathrm{m}^{3}$. In the whole set of data Pousada (1984) shows that, for samples having the same compaction history, the macrostructural plastic strain decreases when the applied vertical stress increases. This expresses the general fact that microstructural swelling has less effect on a dense than on a loose macrostructure. As a consequence, $f_{\mathrm{D}}$ is taken as decreasing with respect to $p / p_{0}$. A parallel physical reasoning leads to the suggestion that $f_{\mathrm{I}}$ should be an increasing function of $p / p_{0}$. Both functions are schematised in Fig. 4.

The variation of volumetric strain with suction for $\sigma_{\mathrm{v}}=0.1 \mathrm{MPa}$ is shown in Fig. 5. In the first cycle, irreversible vertical compression strain develops during the drying stage and additional irreversible swelling strain during the wetting stage. As the cycles go on, strain accumulation reduces. At the end of the test, a net swelling strain of $1.3 \%$ was measured. On the other hand, Dif and Bluemel (1991) presented results on Hoheneggelsen clay $\left(w_{\mathrm{L}}=44-55 \%, w_{\mathrm{P}}=15-21 \%, w_{\mathrm{opt}}=16.6 \%\right.$ and 


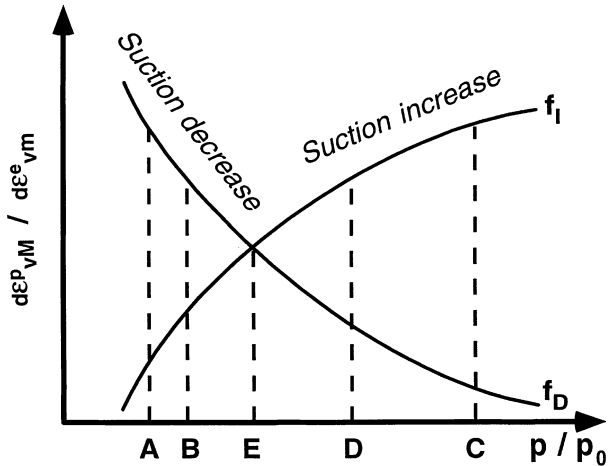

Fig. 4. Typical shape of coupling functions $f_{\mathrm{I}}$ and $f_{\mathrm{D}}$.

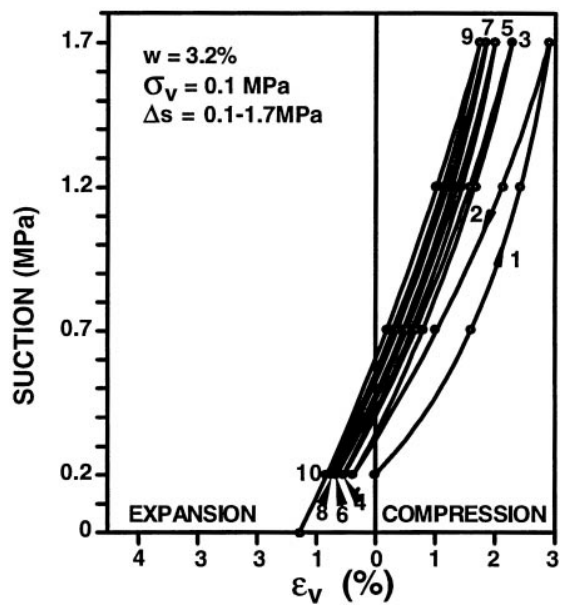

Fig. 5. Accumulation of expansion strain during suction cycles (after Pousada, 1984).

$\gamma_{\text {Dopt }}=16.7 \mathrm{kN} / \mathrm{m}^{3}$ ) in which a global contraction of the material is observed when the sample is alternately soaked and air-dried under a vertical stress of $200 \mathrm{kPa}$. The evolution of the axial deformation measured during the test and the schematic associated strain-suction path are depicted in Fig. 6. These results evidence that the amount of elastoplastic macrostructural strain build-up during a cycle in which SI and SD are alternately activated depends on the level of stress and the history of hydration. Moreover, this amount seems to tend to a stationary value when a large number of cycles is performed. The use of the coupling functions previously defined provides a simple explanation for this phenomenon. When cycles of suction are performed at constant $p$ and low
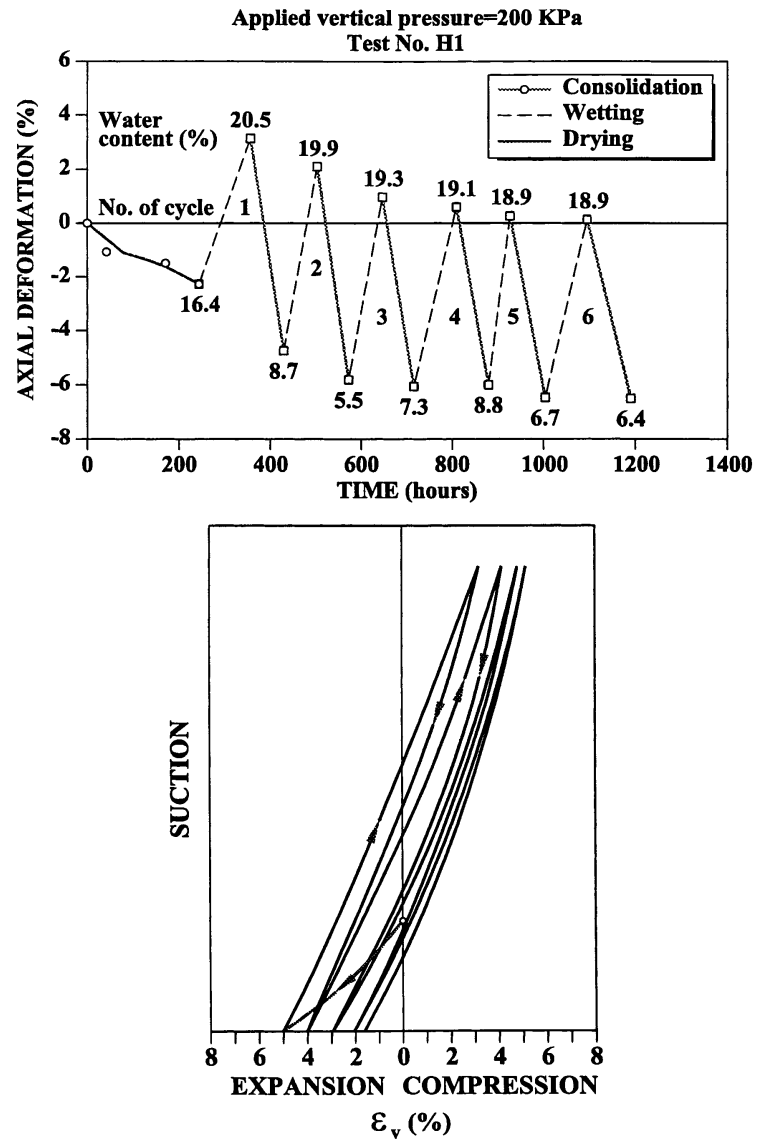

Fig. 6. Accumulation of compression strain during suction cycles (modified after Dif and Bluemel, 1991).

confining stress (point $\mathrm{A}$ in Fig. 4), that is on the left side of point $\mathrm{E}$, the plastic strain during wetting predominates over the plastic strain during drying, leading to a global expansion of the material at the end of a cycle. As a result of the increase in volume, LC shrinks and the value of $p / p_{0}$ increases. The following cycle will then begin at a point such as $\mathrm{B}$, and the global expansion at the end of the new cycle is lower than in the previous one. If a large number of cycles is performed, LC will move in such a way that the ratio $p / p_{0}$ ends up at point E. Starting from the right side of the equilibrium point (point $\mathrm{C}$ ), the opposite mechanism occurs and the material contracts. LC swells and $p / p_{0}$ decreases (point D). Again, after a large number of cycles, LC will find a location which brings $p / p_{0}$ at $\mathrm{E}$. $\mathrm{E}$ then appears to be the equilibrium 
point for a large number of suction cycles. At this point, macrostructural plastic strains still develop, but since they compensate themselves during the suction increase and suction decrease step, no global changes in volume can be observed.

\subsection{Additional experimental evidence}

In highly active clays, microstructure expansion may result in invasion of the macroporosity, when the macrostructure is soft enough. This fact has been reported for instance by Komine and Ogata (1994) in the case of the hydration of a bentonite plug in a radioactive waste disposal. This mechanism causes a decrease in the macrostructural void ratio while the microstructural void ratio expands. Defining the increment of volumetric macrostructural strain $\mathrm{d} \epsilon_{\mathrm{vm}}$ and the increment of volumetric microstructural strain $\mathrm{d} \epsilon_{\mathrm{vM}}$ by:

$\mathrm{d} \epsilon_{\mathrm{vM}}=\mathrm{d} e_{\mathrm{M}} /\left(1+e_{\mathrm{M}}\right)$

$\mathrm{d} \epsilon_{\mathrm{vm}}=\mathrm{d} e_{\mathrm{m}} /\left(1+e_{\mathrm{m}}\right)$

where $e_{\mathrm{M}}$ and $e_{\mathrm{m}}$ are respectively the macrostructural and microstructural void ratio, macropore invasion corresponds to the situation when $\mathrm{d} \epsilon_{\mathrm{vm}}$ is positive and $\mathrm{d} \epsilon_{\mathrm{vM}}$ negative. Since most of the macrostructural strain is of plastic origin, the ratio $\mathrm{d} \epsilon_{\mathrm{vM}}^{\mathrm{p}} / \mathrm{d} \epsilon_{\mathrm{vm}}^{\mathrm{p}}=f_{\mathrm{D}}$ has to be negative at high values of $p / p_{0}$ for highly expansive materials.

On the other hand, data existing in the literature evidence that strong drying induces a reduction in the plasticity index (Mitchell, 1993), a decrease in the swelling pressure of the material (Al-Homoud et al., 1995) and an increase in the hydraulic conductivity (Shear et al., 1992). Although no definitive conclusions can be derived from the present knowledge, these experimental facts are consistent with the build-up of aggregates during drying, accompanied by an increase in the macroporosity. As for the formulation of macropore invasion mechanism, the increase of macroporosity can be introduced into the model by considering negative values for the function $f_{\mathrm{I}}$ at low ratio $p / p_{0}$.

\subsection{Summary of micro-macrostructure interaction mechanisms}

The distinct micro-macropore interaction mechanisms and their representation by the coupling functions $f_{\mathrm{I}}$ and $f_{\mathrm{D}}$ are summarised in Fig. 7. By this simple formalism, global expansion of the material during application of suction cycles at low confining stress, global contraction of the material during application of suction cycles at high confining stress, attenuation of volume changes with the progression of the cycles, macropore invasion by material from the microstructure and development of macroporosity during strong drying can he represented.

\section{Mathematical formulation}

Volumetric stress-strain behaviour is considered for the microstructure, while macrostructure behaviour includes volumetric and deviatoric effects. In a first part, only the volumetric part of the model is described. Extension to deviatoric stress state is presented afterwards.

\subsection{Isotropic stress states}

For the sake of simplicity, local hydraulic and mechanical equilibrium is assumed between the two levels of structure. A unique set of stress variables is then considered in the model: the net stresses and the total suction, defined as the water potential existing in the macropores. When tran-

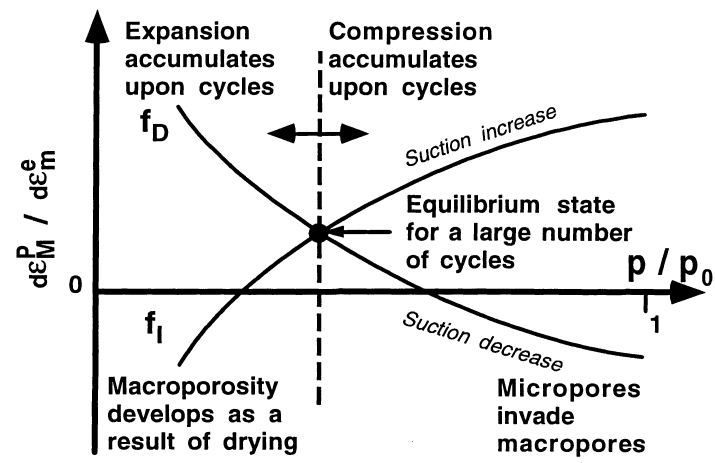

Fig. 7. Summary of micro-macropore interaction mechanisms. 
sient hydro-mechanical response is focused, distinctions between microstructural and macrostructural suction as well as matrix and osmotic suction at both levels have to be considered in order to take into account the different exchange rates of water and ions due to pressure and concentration gradients between aggregates and soil macrostructure. It is not within the scope of the paper to deal with this problem, and more details can be found in Alonso (1998). Particularly, it is shown that secondary swelling can be at least qualitatively reproduced by such an approach.

In mineralogically active soils, micropore size may be of the order of double layer thickness and remains generally saturated. In materials with less pronounced double porosity, microstructural aggregates may exist in unsaturated state. To introduce some generality while maintaining a relatively simple approach, the behaviour of the microstructure is formalised by means of an effective stress concept generalised for unsaturated conditions. A microstructural effective mean stress is defined by:

$\hat{p}=p+S_{\mathrm{r}}^{\alpha} s$

where $S_{\mathrm{r}}$ is the degree of saturation. When $\alpha=0$, saturated effective stress is recovered. When $\alpha=1$, $\hat{p}$ corresponds to Bishop mean stress. At the stress state $\hat{p}_{\mathrm{i}}$, the equation of NL is: $\hat{p}=\hat{p}_{\mathrm{i}}$. Its inclination in the $p-s$ plane may then vary depending on the stress-suction state, the value of $\alpha$ and the retention curve of the material.

Two alternative constitutive laws are considered for the microstructural volumetric elastic strain:

$\mathrm{d} \epsilon_{\mathrm{vm}}^{\mathrm{e}}=\frac{\mathrm{d} \hat{p}}{K_{\mathrm{m}}} \quad$ with $K_{\mathrm{m}}=\frac{\mathrm{e}^{-\alpha_{\mathrm{m}} \hat{p}}}{\beta_{\mathrm{m}}}$ or $K_{\mathrm{m}}=\frac{\left(1+e_{\mathrm{m}}\right) \hat{p}}{\kappa_{\mathrm{m}}}$

depending of the type of expansion to model. As in BBM, the macrostructural volumetric elastic strain is expressed as a function of mean net stress and suction:

$$
\begin{aligned}
& \mathrm{d} \epsilon_{\mathrm{vM}}^{\mathrm{e}}=\frac{\mathrm{d} p}{K_{\mathrm{t}}}+\frac{\mathrm{d} s}{K_{\mathrm{s}}} \quad \text { with } K_{\mathrm{t}}=\frac{\left(1+e_{\mathrm{M}}\right) p}{\kappa} \\
& \text { and } K_{\mathrm{s}}=\frac{\left(1+e_{\mathrm{M}}\right)\left(s+p_{\mathrm{atm}}\right)}{\kappa_{\mathrm{s}}}
\end{aligned}
$$

where $p_{\text {atm }}$ is the atmospheric pressure, $\kappa$ the slope of the unloading-reloading line in an $e-\ln (p)$ diagram and $\kappa_{\mathrm{s}}$ the slope of the reversible wettingdrying line in an $e-\ln (s)$ diagram. The total volumetric elastic strain $\mathrm{d} \epsilon_{\mathrm{v}}^{\mathrm{e}}$ is the sum of $\mathrm{d} \epsilon_{\mathrm{vm}}^{\mathrm{e}}$ and $\mathrm{d} \epsilon_{\mathrm{vM}}^{\mathrm{e}}$.

SI and SD yield loci are taken parallel to the NL and are expressed respectively by:

$\hat{p}-s_{\mathrm{i}}=0$ and $s_{0}-\hat{p}=0$

where $s_{\mathrm{i}}$ and $s_{0}$ are hardening parameters. As in $\mathrm{BBM}$, the variation of preconsolidation pressure $p_{0}$ with suction, which defines the shape of the LC yield surface in the $p-s$ plane, reads:

$p_{0}=p_{\mathrm{c}}\left(\frac{p_{0}^{*}}{p_{\mathrm{c}}}\right)^{\frac{\lambda(0)-\kappa}{\lambda(s)-\kappa}}$

with $\lambda(s)=\lambda(0)\left[r+(1-r) \mathrm{e}^{-\beta s}\right]$

where $\lambda(s)$ is the slope of the virgin consolidation line at suction $s, \lambda(0)$ is the slope of the virgin saturated consolidation line, $p_{0}^{*}$ the saturated preconsolidation pressure, $p_{\mathrm{c}}$ a reference stress, $r$ and $\beta$ model parameters.

Two internal variables are defined for the hardening laws of SI, SD and LC. SI and SD hardening is governed by $\mathrm{d} \alpha_{1}=\mathrm{d} \epsilon_{\mathrm{vSI}}^{\mathrm{p}}+\mathrm{d} \epsilon_{\mathrm{vSD}}^{\mathrm{p}}$ where $\mathrm{d} \epsilon_{\mathrm{vSI}}^{\mathrm{p}}$ and $\mathrm{d} \epsilon_{\mathrm{vSD}}^{\mathrm{p}}$ are the volumetric plastic strains due to activation of respectively SI and SD. LC hardening is governed by $\mathrm{d} \alpha_{2}=\mathrm{d} \epsilon_{\mathrm{vSI}}^{\mathrm{p}}+\mathrm{d} \epsilon_{\mathrm{vSD}}^{\mathrm{p}}+\mathrm{d} \epsilon_{\mathrm{vLC}}^{\mathrm{p}}$ where $\mathrm{d} \epsilon_{\mathrm{vLC}}^{\mathrm{p}}$ is the volumetric plastic strain due to activation of LC. Hardening laws read:

$\mathrm{d} s_{\mathrm{i}}=\frac{K_{\mathrm{m}} \mathrm{d} \alpha_{1}}{f}=\mathrm{d} s_{0}$ and $\frac{\mathrm{d} p_{0}^{*}}{p_{0}^{*}}=\frac{\left(1+e_{\mathrm{M}}\right) \mathrm{d} \alpha_{2}}{\lambda(0)-\kappa}$.

The function $f$ corresponds to the micro-macrostructural coupling function in use, that is $f=f_{\mathrm{I}}$ if SI is active and $f=f_{\mathrm{D}}$ if $\mathrm{SD}$ is active. These hardening laws provide a unidirectional coupling in the sense that LC moves when SI or SD is activated, while activation of LC does not produce any hardening of SI and SD. When more experimental data become available, other types of coupling can be contemplated. Any suitable mathematical function for $f_{\mathrm{I}}$ and $f_{\mathrm{D}}$, consistent with the physical ideas presented in Fig. 7, could be selected. A possible choice is given by the 
following functions:

$f_{\mathrm{I}}=f_{\mathrm{I} 0}+f_{\mathrm{I} 1}\left(p / p_{0}\right)^{n_{\mathrm{I}}}$ and $f_{\mathrm{D}}=f_{\mathrm{D} 0}+f_{\mathrm{D} 1}\left(1-p / p_{0}\right)^{n_{\mathrm{D}}}$.

\subsection{Extension to triaxial stress states}

For triaxial stress conditions, the macrostructural elastic strain has an additional distortion component given by the classical isotropic elasticity:

$\mathrm{d} \epsilon_{\mathrm{qM}}^{\mathrm{e}}=\frac{\mathrm{d} q}{3 G} \quad$ with $G=\frac{3(1-2 v)}{2(1+v)} K_{\mathrm{t}}$

where $q$ is the deviatoric stress, $K_{\mathrm{t}}$ the bulk modulus in front of stress changes, $G$ the shear modulus and $v$ Poisson's ratio, taken constant in the model. Microstructural elastic strain remains volumetric.

In a $p-q-s$ diagram (see Fig. 1), LC yield locus is expressed by:

$q^{2}-M^{2}\left(p+k_{\mathrm{s}} s\right)\left(p_{0}-p\right)=0$.

$M$ is the slope of the critical state line, $k_{\mathrm{s}}$ a parameter describing the increase of cohesion with suction, and $p_{0}$ is given by Eq. (5). SI and SD equations remain unchanged.

Since the hardening laws depend only on the volumetric plastic strain, no significant changes are introduced with respect to the formulation for isotopic stress state. The only difference comes from the fact that, when a deviatoric stress exists, the distance from the stress state to the LC yield locus cannot be expressed by $p / p_{0}$. In this case, the measure adopted for the distance introduced in the functions $f_{\mathrm{I}}$ and $f_{\mathrm{D}}$ reads $p^{*} / p_{0}$, where:

$p^{*}=p+\frac{q^{2}}{M^{2}\left(p+k_{\mathrm{s}} s\right)}$.

Non-associativity is considered for all the yield surfaces. The expression for LC flow rule is:

$\gamma q^{2}-M^{2}\left(p+k_{\mathrm{s}} s\right)\left(p_{0}-p\right)=0$

where $\gamma$ is adopted in order to predict zero lateral strain for $K_{0}$ saturated loading (Ohmaki, 1982).

When SI or SD are reached at a mean net stress $p$, a deviator $q$ and a suction $s$, the direction of plastic strain is given by the flow rule at the image point on the LC yield surface. The co-ordinates of the image point $\left(p^{*}, q^{*}, s^{*}\right)$ are given by:

$p^{*}=\frac{\eta^{2} k_{\mathrm{s}} s-M^{2} p_{0}}{\eta^{2 \prime}+M^{2}}, q^{*}=\eta\left(p+k_{\mathrm{s}} s\right), s^{*}=s$

where $\eta=\frac{q}{p+k_{\mathrm{s}} s}$.

\section{Model performance}

\subsection{Simulation of suction cycles}

Synthetic results of the effect of drying-wetting cycles on strain accumulation have been obtained by using BExM with typical properties of a clay. In Fig. 8, evolution of the total volumetric strain with suction is depicted for an initial value of $p / p_{0}$ located at the left-hand side of the equilibrium point. As discussed in Section 3.3, since the value $f_{\mathrm{D}}$ function is higher than the value $f_{\mathrm{I}}$ function (see Fig. 9), expansion strains accumulate, which corresponds to the case of the figure presented by Pousada (1984).

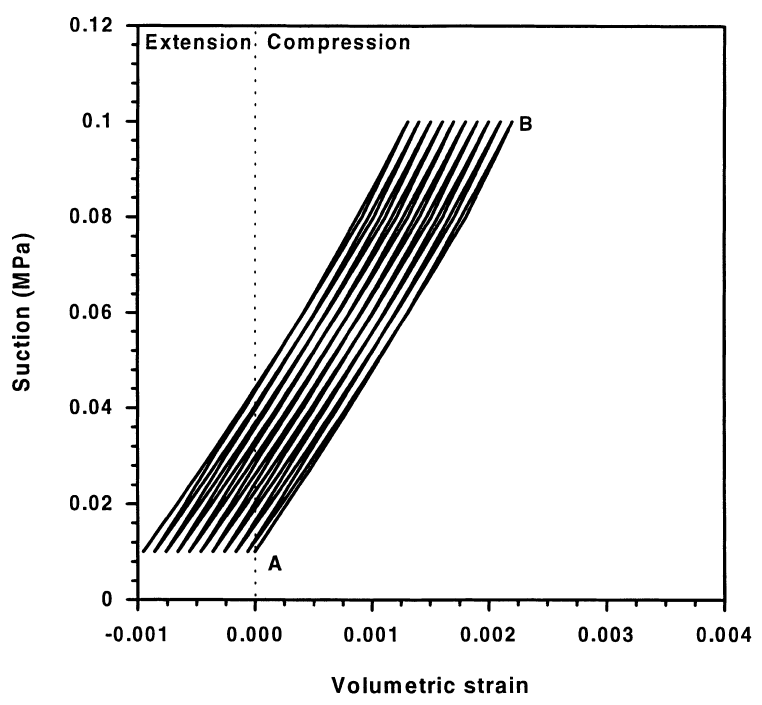

Fig. 8. Model results: strain evolution during suction cycles. 


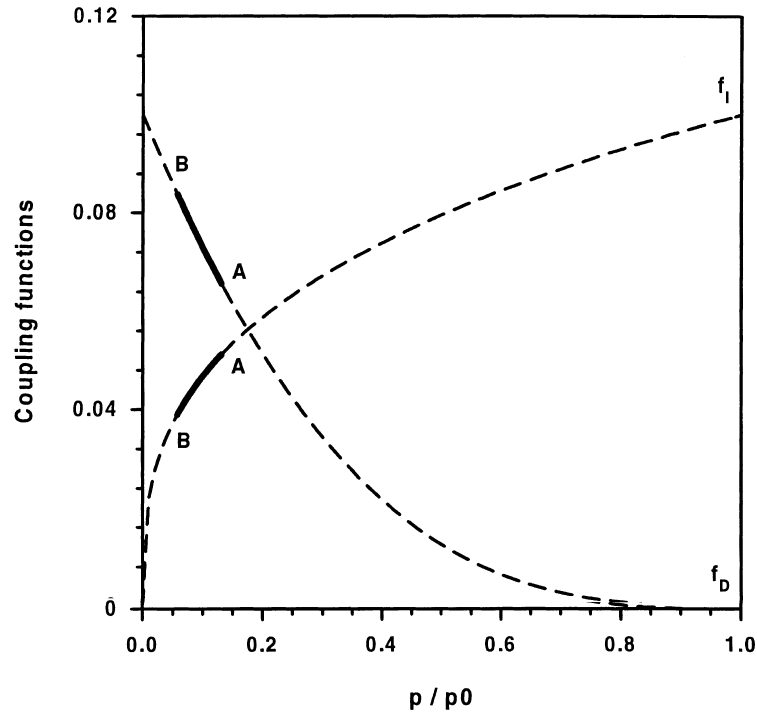

Fig. 9. Model results: values adopted by the coupling functions during one suction cycle.

\subsection{Simulation of oedometer suction-controlled tests on compacted pellets of Boom clay}

Alonso et al. (1995) presented five tests performed in a suction-controlled oedoemeter apparatus at different vertical loads $(20 \mathrm{kPa}, 50 \mathrm{kPa}$, $100 \mathrm{kPa}, 200 \mathrm{kPa}$ and $400 \mathrm{kPa})$. The specimens

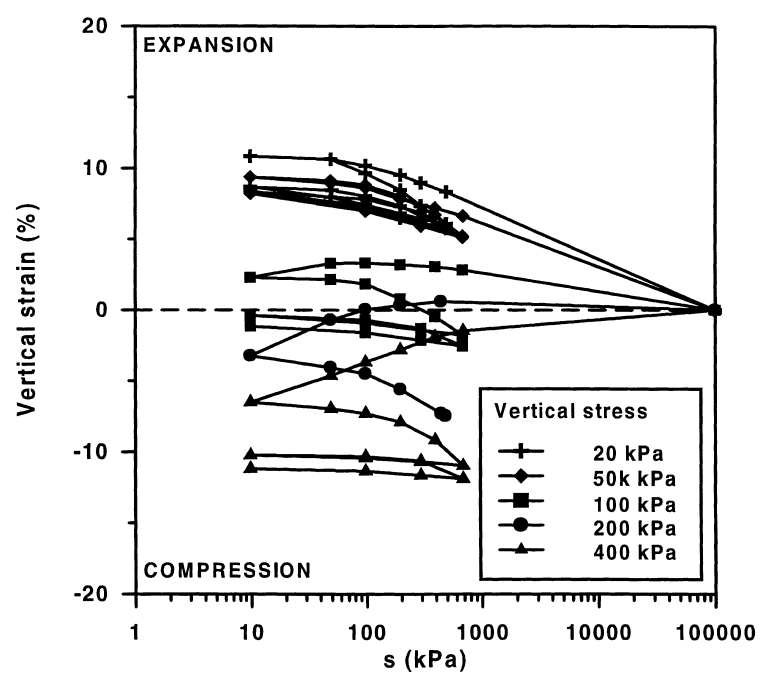

Fig. 10. Suction-controlled oedometer tests on compacted pellets of Boom clay.

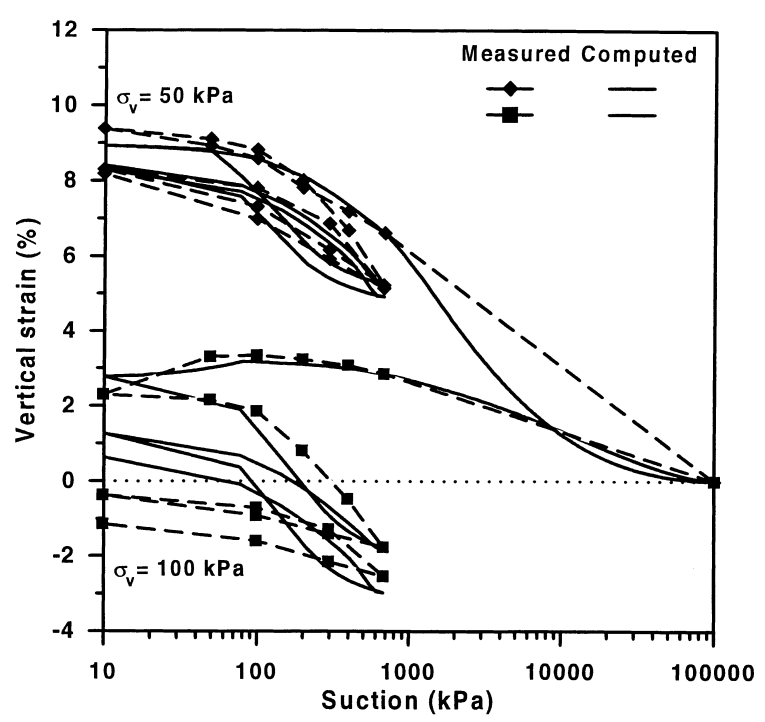

Fig. 11. Modelling of oedometer suction-controlled tests on compacted pellets of Boom clay.

were prepared by compacting to an overall dry density of $14 \mathrm{kN} / \mathrm{m}^{3}$ highly compacted pellets of Boom clay $\left(w_{\mathrm{L}}=55.9 \%, w_{\mathrm{P}}=29.2 \% \quad\right.$ and $\left.I_{\mathrm{P}}=26.7 \%\right)$. The pellets have an initial water content of $3 \%$, an initial dry density of $20 \mathrm{kN} / \mathrm{m}^{3}$, an average size of $2 \mathrm{~mm}$ and act as expansive aggregates. The samples were placed in a dry state

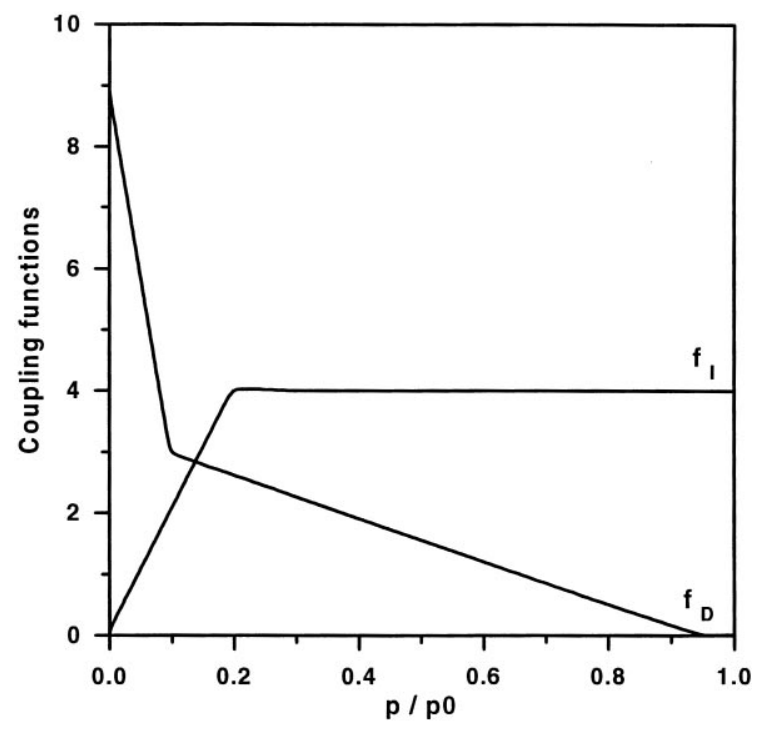

Fig. 12. Coupling functions used in the simulation. 
Table 1

Material parameters used in the simulation

\begin{tabular}{|c|c|c|c|c|c|c|c|c|c|c|c|}
\hline$\alpha$ & $\kappa_{\mathrm{m}}$ & $\kappa$ & $\kappa_{\mathrm{s}}$ & $\lambda(0)$ & $r$ & $\beta\left(\mathrm{kPa}^{-1}\right)$ & $p_{\mathrm{c}}(\mathrm{kPa})$ & $v$ & $k_{\mathrm{s}}$ & $M$ & $S_{\mathrm{r}}$ \\
\hline 2 & 0.01 & 0.045 & $\mathrm{a}$ & 0.016 & 0.564 & 0.00544 & 42 & 0.3 & 0.0073 & 1 & $\mathrm{~b}$ \\
\hline
\end{tabular}

${ }^{\mathrm{a}} \mathrm{A}$ dependence on mean net stress is introduced: $\kappa_{\mathrm{s}}(p)=\max \{0.005,0.0398-0.00745 \log [p(\mathrm{kPa})]\}$.

${ }^{\mathrm{b}} S_{\mathrm{r}}$ is computed from the retention curve: $S_{\mathrm{r}}(s)=0.08+0.92\{1.22-0.22 \log [s(\mathrm{kPa})]\}$.

Table 2

History variables considered in the simulation

\begin{tabular}{llllll}
\hline Test & $e_{\mathrm{m}}$ & $e_{\mathrm{M}}$ & $p_{0}^{*}(\mathrm{kPa})$ & $s_{\mathrm{i}}-s_{0}(\mathrm{kPa})$ & $s_{0}(\mathrm{kPa})^{\mathrm{a}}$ \\
\hline$\sigma_{\mathrm{v}}=50 \mathrm{kPa}$ & 0.285 & 0.932 & 450 & 25 & 3660 \\
$\sigma_{\mathrm{v}}=100 \mathrm{kPa}$ & 0.285 & 0.932 & 450 & 25 & 3895 \\
\hline
\end{tabular}

${ }^{\mathrm{a}} s_{0}$ is fixed by the initial stress state.

inside the apparatus and wetted up to a suction of $10 \mathrm{kPa}$. Afterwards, cycles of suction between $10 \mathrm{kPa}$ and $600 \mathrm{kPa}$ were applied. The value of vertical strain measured in the five tests shows a progressive transition from swelling to collapse as the vertical applied load increases (see Fig. 10). This general trend is consistent with the elastoplastic framework developed in this paper. In Fig. 11, comparison between model simulations and experimental results is shown for the test with a vertical load of $50 \mathrm{kPa}$ and $100 \mathrm{kPa}$. The parameters used are presented in Tables 1 and 2 and the coupling functions in Fig. 12. The tests are qualitatively well-reproduced. A satisfactory agreement from a quantitative point of view can also be observed.

\section{Conclusions}

On the basis of experimental evidence, the elastoplastic model proposed by Gens and Alonso (1992) and Alonso et al. (1994) for expansive soils is reviewed in order to include features related to cyclic behaviour on drying-wetting paths with a minimal additional set of parameters. Based on the definition of two coupling functions that express the ratio between the microstructural strain and the resulting macrostructural plastic strain, the model allows a good representation of the phenomena of stress-suction path dependency and swelling-shrinkage fatigue during cycles. It also includes more specific features such as macropore intrusion by expanded microstructure and an increase of macroporosity during strong drying. Comparison with experimental tests performed in a suction-controlled oedometer apparatus shows the ability of the model to capture well the qualitative trends of the data and to adjust them quantitatively in a satisfactory way. Other aspects such as transient response and secondary swelling of expansive clay may be accommodated within the framework described.

\section{Acknowledgement}

The support of the DGICYT through research grant PB95-0771 is gratefully acknowledged.

\section{References}

Al-Homoud, A.S., Basma, A.A., Malkawi, A.I., Al Bashabsheh, M.A., 1995. Cyclic swelling behavior of clays. J. Geotech. Eng. 121 (7), 562-565.

Alonso, E.E., 1998. Keynote Lecture, Proc. 2nd Int. Conf. on Unsaturated Soils, International Academic, Beijing, China, in press.

Alonso, E.E., Gens, A., Hight, D.W., 1987. Special problem soils, general report, Proc. 9th ECSMFE Vol. 3. Balkema, Dublin, pp. 1087-1146.

Alonso, E.E., Gens, A., Josa, A., 1990. A constitutive model for partially saturated soils. Géotechnique 40 (3), 405-430. 
Alonso, E.E., Gens, A., Yuk Gehling, W.Y., 1994. Elastoplastic model for unsaturated expansive soils, Proc. 3rd European Conf. on Numerical Methods in Geotechnical EngineeringBalkema, Manchester, pp. 11-18.

Alonso, E.E., Lloret, A., Gens, A., Yang, D.Q., 1995. Experimental behaviour of higly expansive double-structure clayProc. 1st Int. Conf. on Unsaturated Soils Vol. 1. Balkema, Paris, pp. 11-16.

Brackley, I.J., 1973. Swell pressure and free swell in compacted clay, Proc. 3rd Int. Conf. on Expansive Soils Vol. 1. Academic Press, Haifa, pp. 169-176.

Dif, A.E., Bluemel, W.F., 1991. Expansive soils under cyclic drying and wetting, technical note. Geotech. Testing J. 14 (1), 96-102.

Gens, A., Alonso, E.E., 1992. A framework for the behaviour of unsaturated expansive clays. Can. Geotech. J. 29, 1013-1032.

Justo, J.L., Delgado, A., Ruiz, J., 1984. The influence of stresspath in the collapse-swelling of soils at the laboratory, Proc. 5th Int. Conf. on Expansive Soils, Institution of Engineers of Australia, Adelaide, pp. 67-71.

Komine, H., Ogata, N., 1994. Experimental study on swelling characteristics of compacted bentonite. Can. Geotech. J. $31,478-490$.
Komornik, A., Zeitlen, J.G., 1965. An apparatus for measuring lateral soil swelling pressure in the laboratory, Proc. 6th ICMSFE Vol. 1. Toronto University Press, Montreal, pp. $278-281$.

Mitchell, J.K., 1993. Fundamentals of Soil Behavior. 2nd edn., Wiley, New York.

Ohmaki, S., 1982. Stress-strain behaviour of anisotropically, normally consolidated cohesive soil, Proc. 5th Int. Symp. on Numerical Models in Geomechanics, Balkema, Zurich, pp. 250-269.

Pousada, E., 1984. Deformabilidad de arcillas expansivas bajo succión controlada. Doctoral Thesis, Universidad Politecnica de Madrid, Spain.

Romero, E., 1999. Characterization and thermo-hydro-mechanical behaviour of unsaturated Boom clay: an experimental study. Doctoral Thesis, Universitat Politecnica de Catalunya, Barcelona. Spain, in preparation.

Roscoe, K.H., Burland, J.B., 1968. On the generalized stressstrain behaviour of the 'wet' clay. In: Heyman, J., Leckie, F.A. (Eds.), Engineering Plasticity, Cambridge University Press, Cambridge, pp. 535-609.

Shear, D.L., Olsen, H.W., Welson, K.R., 1992. Effect of desiccation on the hydraulic conductivity versus void ratio relationship for a natural clay. Transport. Res. Rec. 1369, 130-135. 